\title{
U-Shaped Relationship Between Left Atrium Size on Echocardiography and 1-Year Recurrence of Atrial Fibrillation After Radiofrequency Catheter Ablation
}

\author{
- Prognostic Value Study -
}

\author{
Qiqi Wang, MD; Chengui Zhuo, BSc; Yunpeng Shang, MD; Jianqiang Zhao, BSc; \\ Nan Chen, BSc; Ning Lv; Yuan Huang, MD, PhD; Liangrong Zheng, MD; \\ Jiangtao Lai, MD; Jie Han, MD; Zheyue Shu, BSc
}

\begin{abstract}
Background: The larger the left atrium anteroposterior dimension (LAD) and left atrium volume (LAV), the stronger the association with recurrent atrial fibrillation (AF) after radiofrequency catheter ablation (RFCA). Patients with a smaller left atrium (LA) size, however, also have increased AF recurrence.
\end{abstract}

\begin{abstract}
Methods and Results: In 521 patients, routine 48-h Holter electrocardiogram and echocardiography were obtained at each outpatient visit every 3 months for 12 months. On multivariate analysis, AF type, LAD, and LAV calculated using the ellipsoid model/body surface area (LAVe/BSA) were independent predictors of $A F$ recurrence. Patients were divided into 7 groups at $0.4-\mathrm{cm}$ increments of $L A D: \leq 3 \mathrm{~cm}, L A D \leq 3 \mathrm{~cm}, 3.0<L A D \leq 3.4 \mathrm{~cm}, 3.4<L A D \leq 3.8 \mathrm{~cm}, 3.8<L A D \leq 4.2 \mathrm{~cm}, 4.2<L A D \leq 4.6 \mathrm{~cm}, 4.6<L A D \leq 5.0 \mathrm{~cm}$; and $L A D>5.0 \mathrm{~cm}$. Compared with the 3.4-3.8-cm group, the adjusted HR were 3.88 (95\% Cl: 2.02-7.46, $\mathrm{P}<0.001), 1.03$ (95\% Cl: 0.50-2.12, $\mathrm{P}=0.939)$, 0.96 (95\% Cl: 0.52-1.77, P=0.901), 1.36 (95\% Cl: 0.72-2.57, P=0.347), 3.04 (95\% Cl: 1.67-5.53, P<0.001), and 4.07 (95\% Cl: 1.93-8.60, $\mathrm{P}<0.001$ ), respectively. Similarly, we divided LAVe/BSA into 8 groups and also observed a U-shaped curve for AF recurrence.
\end{abstract}

Conclusions: Both larger and smaller LAD and LAVe/BSA were associated with a higher risk of AF recurrence 1 year after RFCA. The association of LA size and AF recurrence after RFCA is represented by a U-shaped curve.

Key Words: Atrial fibrillation recurrence; Echocardiography; Left atrium anteroposterior dimension; Left atrium volume; Radiofrequency catheter ablation

A trial fibrillation (AF) is the most common sustained arrhythmia occurring in the general population. ${ }^{1}$ In China, AF has an estimated prevalence of $0.77 \%$ with an age-adjusted rate of 0.61 , thus suggesting that approximately 8 million adults in China have AF. ${ }^{2}$ Radiofrequency catheter ablation (RFCA), which targets the isolation of pulmonary veins (PV), has emerged as a cornerstone in the treatment of AF. In the 2014 American Heart Association/American College of Cardiology/Heart Rhythm Society (AHA/ACC/HRS) Atrial Fibrillation Guidelines, catheter ablation is a class I recommendation supported with level A evidence, ${ }^{3}$ but not all patients maintain sinus rhythm after catheter ablation, and there can be both early and late relapses to AF in many patients. ${ }^{4}$ Thus, evaluation of the proportion of recurrences after catheter ablation is warranted.

Left atrial (LA) enlargement is associated with atrial remodeling, which is related to AF recurrence after RFCA. ${ }^{5}$ The LA anteroposterior dimension (LAD) and LA volume (LAV) are widely used in clinical practice and research as a non-invasive method of determining LA enlargement. There is a linear relationship between LAD and post-ablation AF recurrence: ${ }^{6}$ the larger the LAD, the higher the prevalence of AF recurrence.

In our large clinical practice, we have also found that a larger LA size significantly increases the risk of AF recurrence 1 year after RFCA, which is consistent with the previous studies; compared with the larger LA, however, we found

Received February 25, 2019; revised manuscript received April 25, 2019; accepted May 2, 2019; J-STAGE Advance Publication released online June 8, 2019 Time for primary review: 30 days

Department of Cardiology and Atrial Fibrillation Center (Q.W., C.Z., Y.S., J.Z., N.C., Y.H., L.Z., J.L., J.H.), Department of Pharmacy (N.L.), Department of Hepatobiliary Surgery (Z.S.), First Affiliated Hospital of Zhejiang University, Hangzhou, Zhejiang Province, China

The first two authors contributed equally to this work (Q.W., C.Z.).

Mailing address: Liangrong Zheng, MD, Department of Cardiology and Atrial Fibrillation Center of The First Affiliated Hospital of Zhejiang University, 79 Qingchun Road, Hangzhou, Zhejiang Province, China. E-mail: 1191066@zju.edu.cn

ISSN-1346-9843 All rights are reserved to the Japanese Circulation Society. For permissions, please e-mail: cj@j-circ.or.jp 


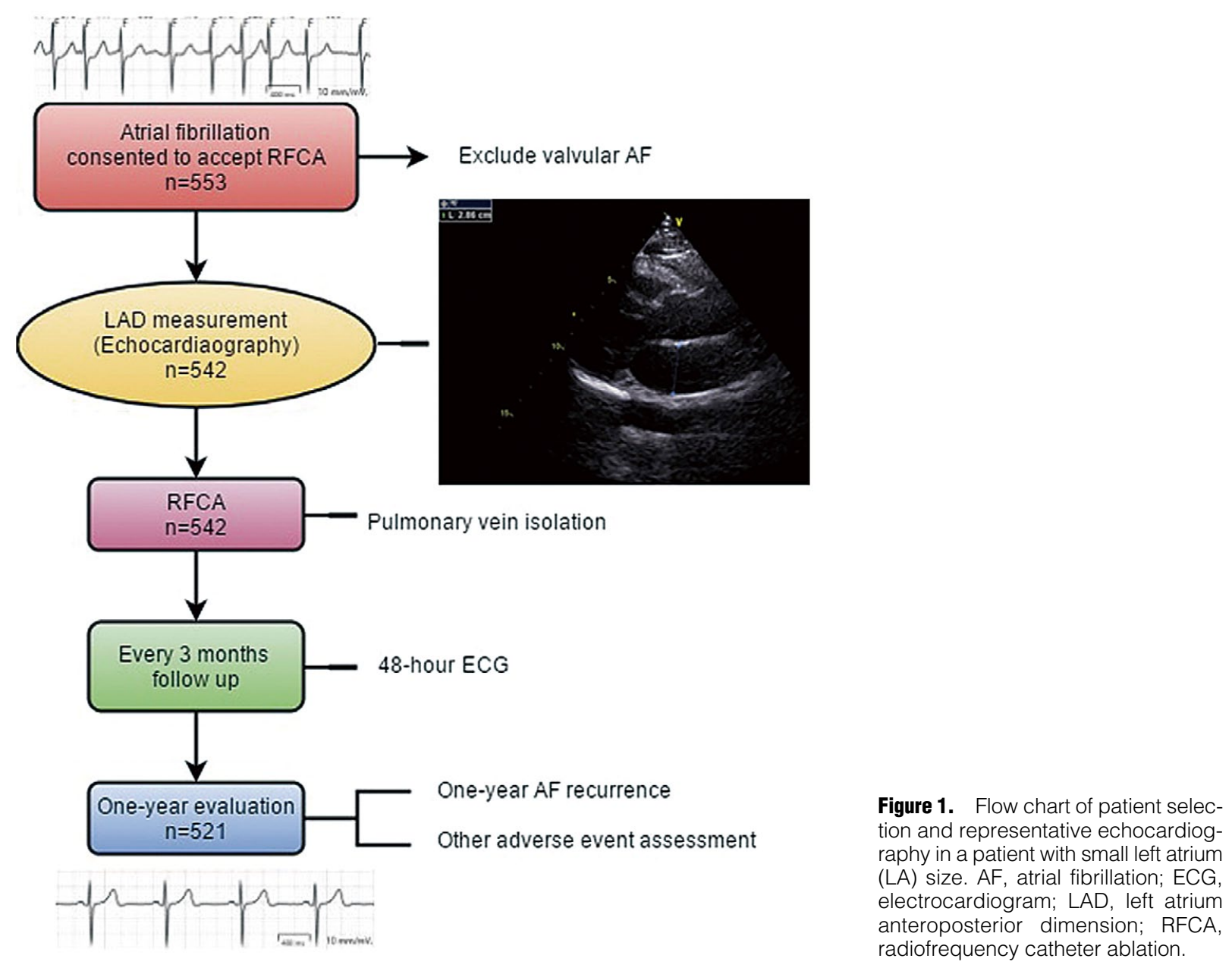

that patients with a smaller LA might also have an increased risk of AF recurrence after catheter ablation. To date, there are no data on the hypothesis that a smaller LAD or LAV may increase the risk of AF recurrence after RFCA. Thus, we evaluated successive ranges of LAD and LAV and the subsequent risk for AF recurrence, in order to clarify the relationship between LA size and the recurrence of AF 1 year after RFCA.

\section{Methods}

\section{Subjects}

This prospective observational study enrolled 542 consecutive patients with paroxysmal or persistent AF who underwent RFCA at the present institution between January 2015 and July 2017.

The inclusion criteria were as follows: $>18$ years of age; non-valvular AF; suitable for catheter ablation; voluntary participation in the study; and signed informed consent.

The exclusion criteria were as follows: New York Heart Association functional classes III and IV; progressive renal insufficiency $\leq 3$ months before ablation; myocardial infarction $(\mathrm{MI})$ or percutaneous coronary intervention $\leq 6$ months before ablation; presence of LA thrombus on transesophageal echocardiography; and the presence of a prosthetic heart valve. Patients with malignancy, autoimmune or inflammatory disease, and severe hepatic and renal dysfunction were excluded before ablation.

Patients were evaluated every 3 months for $\geq 12$ months after the initial ablation procedure (Figure 1). Nineteen patients were lost to follow-up and 2 patients died. The Institutional Review Boards at Zhejiang University (Hangzhou, Zhejiang Province, China) approved this study, and all of the subjects gave written informed consent. The study was registered at http://www.chictr.org.cn (identifier ChiCTR-1800017465).

\section{Echocardiography}

Transthoracic echocardiography was performed by a dedicated sonographer and recorded using a commercially available echocardiographic system (VIVID E9; General Electric, Milwaukee, WI, USA). All of the parameters were obtained in accordance with the American Society of Echocardiography and European Association of Cardiovascular Imaging recommendations. ${ }^{7}$ The LAD (D) was measured from the parasternal long-axis view orthogonal to the aortic root during the left ventricular (LV) end-systole phase (Figure 2). LA transverse (T) and longitudinal (L) dimensions were taken from the apical 4-chamber views using the inner edgeinner edge method. LAV was calculated using the ellipsoid model (LAVe) according to the following formula: ${ }^{7}$ 

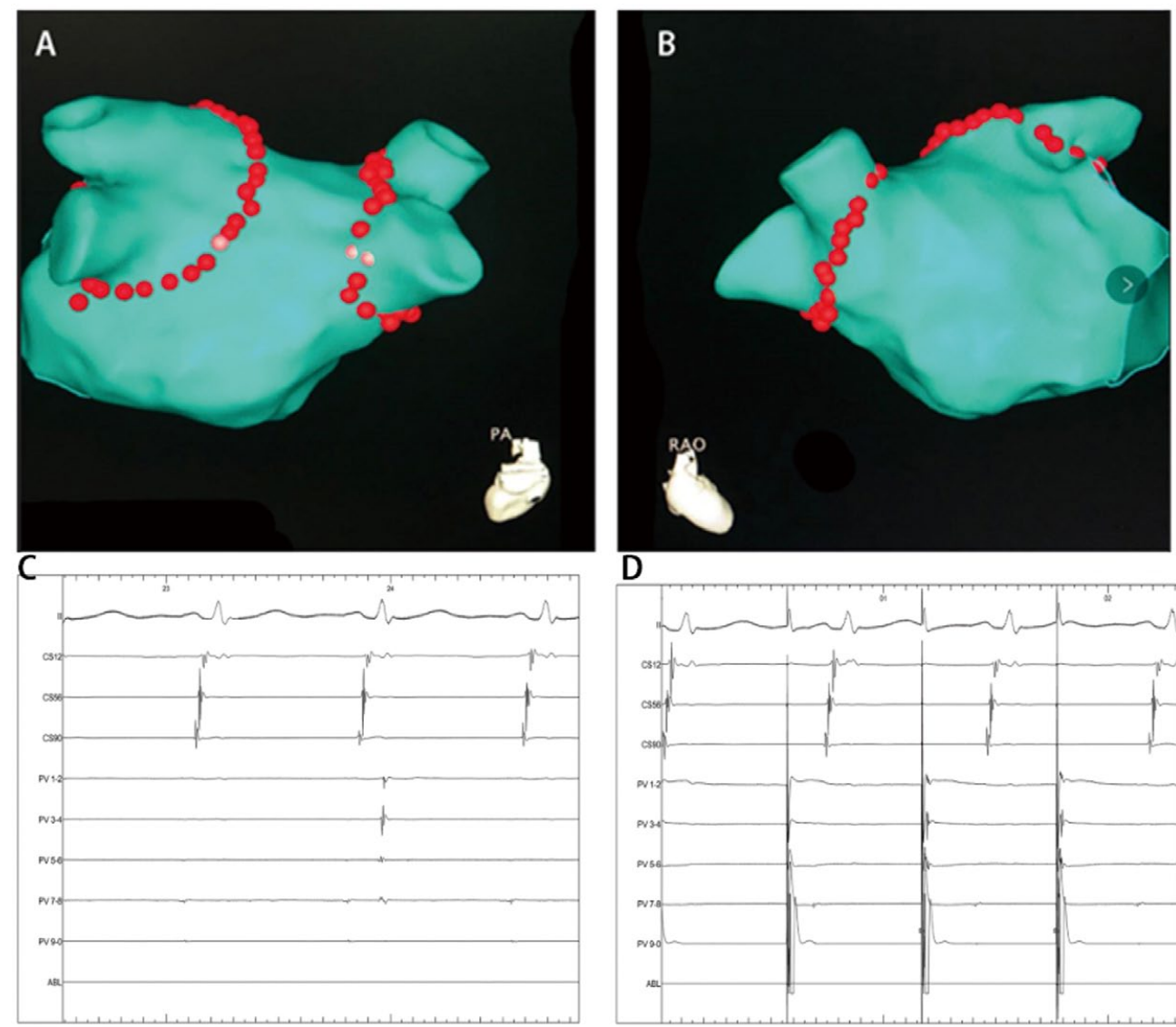

D

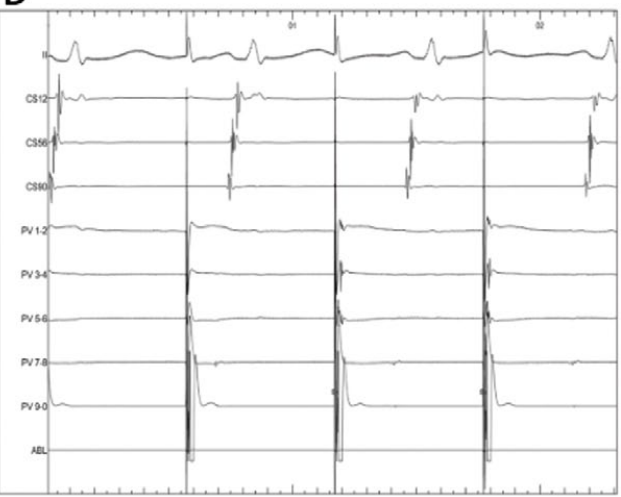

Figure 2. (A) Posteroanterior (PA) and (B) right anterior oblique (RAO) view on CARTO. (C) After pulmonary vein (PV) isolation, there was an automatic potential in the PV that could not conduct to the atrium. (D) After PV isolation, PV activation during pacing was dissociated from atrial activation in all tracings.

\section{$\mathrm{LAVe}=\pi / 6(\mathrm{LAD} \times \mathrm{L} \times \mathrm{T})$}

LV ejection fraction (LVEF) and LV end-diastolic dimension (LVEDD) were measured on M-mode echocardiography on parasternal long-axis imaging during the LV end-diastolic phase. Body surface area (BSA) was calculated using the following formulae:

$$
\begin{aligned}
\text { BSAmen }= & 0.0057 \times \text { height }(\mathrm{cm})+0.0121 \times \text { weight }(\mathrm{kg}) \\
& +0.0882 \text { and } \\
\text { BSA }_{\text {women }}= & 0.0073 \times \text { height }(\mathrm{cm})+0.0127 \times \text { weight }(\mathrm{kg}) \\
& -0.2106 .
\end{aligned}
$$

We used LAVe/BSA as an indicator of LAV for each patient.

\section{Catheter Ablation}

All of the patients discontinued anti-arrhythmic drugs (AAD) for $\geq 5$ half-lives $\geq 3$ months before RFCA. Transesophageal echocardiography was performed 1 day before the procedure to confirm the absence of atrial thrombi. RF-based circumferential PV isolation was performed using an irrigated RF ablation catheter (Thermocool; Biosense Webster, Diamond Bar, CA, USA) with the aid of electroanatomic mapping (Carto 3; Biosense Webster; Figure 2). Complete $\mathrm{PV}$ isolation was performed in all of the patients. Superior vena cava isolation ablation, linear ablation in the LA roof and cavo-tricuspid isthmus ablation were performed at the discretion of the operator. Testing with adenosine was not routinely performed. After complete PV isolation, a minimum waiting time of $30 \mathrm{~min}$ from the last $\mathrm{PV}$ isolation was mandated. Successful ablation was defined as complete elimination of all fragmented signals at the PV ostium, as well as verified exit and entrance block (Figure 2). Entrance block was demonstrated by the absence, or, if present, the dissociation of, electrical activity in the PV antrum. Exit block was confirmed by PV activation during pacing dissociated from atrial activation in all tracings. If AF or other types of sustained atrial tachyarrhythmia persisted after initial ablation, direct current cardioversion was performed to restore sinus rhythm.

\section{Follow-up}

Patients were evaluated every 3 months in the dedicated arrhythmia outpatient clinic for $\geq 12$ months (Figure 1). Routine echocardiograms and 48-h Holter electrocardiograms were obtained at each outpatient visit to demonstrate AF recurrence.

Previous anti-arrhythmic and anti-coagulant therapy was maintained for $\geq 3$ months. Medication was discontinued or reduced at the discretion of the investigator based on $\mathrm{AF}$ recurrence and $\mathrm{CHA}_{2} \mathrm{DS}_{2}-\mathrm{VASc}$ score. The primary 


\begin{tabular}{|c|c|c|c|c|}
\hline Characteristics & $\begin{array}{c}\text { Total } \\
(n=521)\end{array}$ & $\begin{array}{c}\text { Recurrence } \\
(n=128)\end{array}$ & $\begin{array}{c}\text { No recurrence } \\
(n=393)\end{array}$ & P-value \\
\hline Age (years) & $60.2 \pm 9.60$ & $59.3 \pm 9.84$ & $60.5 \pm 9.51$ & 0.596 \\
\hline Male & 327 (62.8) & $85(66.4)$ & $242(61.6)$ & 0.326 \\
\hline BMI $\left(\mathrm{kg} / \mathrm{m}^{2}\right)^{\dagger}$ & $24.5(4.2)$ & $24.4(4.3)$ & $24.5(4.4)$ & 0.401 \\
\hline \multicolumn{5}{|l|}{ Medication use } \\
\hline$\beta$-blocker & $220(42.2)$ & $59(46.1)$ & $161(41.0)$ & 0.308 \\
\hline ACEI/ARB & $154(29.6)$ & $34(26.6)$ & $120(30.5)$ & 0.392 \\
\hline \multicolumn{5}{|l|}{ History } \\
\hline Hypertension & 237 (45.5) & $52(40.6)$ & $185(47.1)$ & 0.203 \\
\hline $\mathrm{DM}$ & $43(8.3)$ & $10(7.8)$ & $33(8.4)$ & 0.835 \\
\hline CVD & $98(18.8)$ & $24(18.8)$ & $74(18.8)$ & 0.984 \\
\hline Type of AF & & & & 0.003 \\
\hline Paroxysmal & $445(85.4)$ & 99 (77.3) & $326(88.0)$ & \\
\hline Persistent & $76(14.6)$ & $29(22.7)$ & $47(12.0)$ & \\
\hline \multicolumn{5}{|l|}{ Echocardiography } \\
\hline LAD (cm) & $4.0 \pm 0.60$ & $4.1 \pm 0.76$ & $3.9 \pm 0.53$ & $<0.001$ \\
\hline $\operatorname{LVEF}(\%)^{\dagger}$ & $65.4(8.0)$ & $66.0(8.0)$ & $65.3(8.5)$ & 0.386 \\
\hline LVD $(\mathrm{cm})$ & $4.7 \pm 0.42$ & $4.7 \pm 0.42$ & $4.7 \pm 0.42$ & 0.947 \\
\hline LAVe/BSA $\left(\mathrm{mL} / \mathrm{m}^{2}\right)$ & $31.81 \pm 11.06$ & $34.81 \pm 13.88$ & $30.79 \pm 9.75$ & $<0.001$ \\
\hline $\mathrm{BSA}\left(\mathrm{m}^{2}\right)$ & $1.81 \pm 0.18$ & $1.82 \pm 0.16$ & $1.80 \pm 0.18$ & 0.297 \\
\hline Anticoagulant & & & & 0.411 \\
\hline Warfarin & $346(66.4)$ & $86(67.2)$ & $260(66.2)$ & \\
\hline Rivaroxaban & $159(30.5)$ & $36(28.1)$ & $123(31.3)$ & \\
\hline Dabigatran & $16(3.1)$ & $6(4.7)$ & $10(2.5)$ & \\
\hline Smoking & $195(37.4)$ & $50(39.1)$ & $145(36.9)$ & 0.660 \\
\hline Alcohol consumption & $157(30.1)$ & $41(32.0)$ & $116(29.5)$ & 0.590 \\
\hline Pacemaker implant & 17 (3.3) & $6(4.7)$ & $11(2.8)$ & 0.296 \\
\hline
\end{tabular}

Data given as mean $\pm \mathrm{SD}$, ${ }^{\dagger}$ median $(\mathrm{IQR})$ or $\mathrm{n}(\%)$. $\mathrm{P}<0.05$ indicates statistical significance. $\mathrm{ACEl}$, angiotensinconverting enzyme inhibitor; $A F$, atrial fibrillation; ARB, angiotensin receptor blocker; BMI, body mass index; $B S A$, body surface area; CVD, cardiovascular disease; DM, diabetes mellitus; LAD, left atrium anteroposterior diameter; LAVe, left atrium volume calculated using the ellipsoid model; LVD, left ventricular diameter; LVEF, left ventricular ejection fraction; RFCA, radiofrequency catheter ablation.

outcome was the 1-year AF recurrence after RFCA. AF recurrence was defined as any documented atrial arrhythmia (AF, atrial flutter, or atrial tachycardia) episode lasting $>30 \mathrm{~s}$ after ablation, excluding a 3-month blanking period.

\section{Statistical Analysis}

For normally distributed data, continuous variables are presented as mean $\pm \mathrm{SD}$, and differences in continuous variables were determined using 1-way ANOVA or Student's t-test. For non-normally distributed data, continuous variables are presented as median (IQR), and differences in continuous variables were determined using the KruskalWallis H- and Mann-Whitney U-tests. Categorical data are presented as $\mathrm{n}(\%)$. Categorical data were compared using the chi-squared or Fisher's exact test. LAD was categorized into $0.4-\mathrm{cm}$ increments in the following manner: $\mathrm{LAD} \leq 3 \mathrm{~cm}, 3.0<\mathrm{LAD} \leq 3.4 \mathrm{~cm}, 3.4<\mathrm{LAD} \leq 3.8 \mathrm{~cm}$, $3.8<\mathrm{LAD} \leq 4.2 \mathrm{~cm}, 4.2<\mathrm{LAD} \leq 4.6 \mathrm{~cm}, 4.6<\mathrm{LAD} \leq 5.0 \mathrm{~cm}$; and LAD $>5.0 \mathrm{~cm}$. LAVe/BSA was categorized into $5-\mathrm{mL} / \mathrm{m}^{2}$ increments in the following manner: LAVe/BSA $\leq 20 \mathrm{~mL} / \mathrm{m}^{2}$, $20<\mathrm{LAVe} / \mathrm{BSA} \leq 25 \mathrm{~mL} / \mathrm{m}^{2}, \quad 25<\mathrm{LAVe} / \mathrm{BSA} \leq 30 \mathrm{~mL} / \mathrm{m}^{2}$, $30<\mathrm{LAVe} / \mathrm{BSA} \leq 35 \mathrm{~mL} / \mathrm{m}^{2}, \quad 35<\mathrm{LAVe} / \mathrm{BSA} \leq 40 \mathrm{~mL} / \mathrm{m}^{2}$, $40<\mathrm{LAVe} / \mathrm{BSA} \leq 45 \mathrm{~mL} / \mathrm{m}^{2}, \quad 45<\mathrm{LAVe} / \mathrm{BSA} \leq 50 \mathrm{~mL} / \mathrm{m}^{2}$, $50 \mathrm{~mL} / \mathrm{m}^{2}<\mathrm{LAVe} / \mathrm{BSA}$. Univariate and multivariate Cox proportional hazard models were used to calculate the hazard ratios (HR) for the different LAD and LAVe/BSA categories to determine the relationship between clinical factors and AF recurrence. The $3.4-3.8-\mathrm{cm}$ and $25-30-\mathrm{mL} / \mathrm{m}^{2}$ groups were used as the reference category. Adjusted HR were estimated after adjusting for age, gender, body mass index (BMI), diabetes mellitus, hypertension, and comorbidities of cardiovascular disease. Two-sided $\mathrm{P}<0.05$ was considered to indicate statistical significance. All statistical analysis was performed using SPSS version 23.00 (SPSS, Chicago, IL, USA).

\section{Results}

\section{Patient Characteristics}

Five hundred and twenty-one consecutive patients were identified for the study cohort and analysis (Table 1). The majority of the cohort were male $(62.8 \%)$, with a mean age of $60.2 \pm 9.60$ years. The smallest and largest LAD was $2.76 \mathrm{~cm}$ and $5.58 \mathrm{~cm}$, respectively. Ninety-eight patients $(18.8 \%)$ had cardiovascular disease, $237(45.5 \%)$ had hypertension, and $76(14.6 \%)$ had persistent AF. Of the 521 patients, $11(2.1 \%)$ underwent a second ablation procedure because of recurrent AF. After the second procedure, 3 patients had recurrent AF. Warfarin was the most frequently used anticoagulant before and after the first 3 months of RFCA. Three hundred and ninety-three patients (75.4\%) in the entire group were free of AF recurrence 1 year after RFCA. 


\begin{tabular}{|c|c|c|c|c|c|c|c|c|}
\hline LAD (cm) & $\begin{array}{c}\leq 3.0 \\
(n=33)\end{array}$ & $\begin{array}{c}3.0<\text { LAD } \\
\leq 3.4 \\
(n=70)\end{array}$ & $\begin{array}{c}3.4<\text { LAD } \\
\leq 3.8 \\
(n=116)\end{array}$ & $\begin{array}{c}3.8<\text { LAD } \\
\leq 4.2 \\
(n=142)\end{array}$ & $\begin{array}{c}4.2<\text { LAD } \\
\leq 4.6 \\
(n=85)\end{array}$ & $\begin{array}{c}4.6<L A D \\
\leq 5.0 \\
(n=55)\end{array}$ & $\begin{array}{c}>5.0 \\
(n=20)\end{array}$ & P-value \\
\hline Recurrence & 17 (51.6) & $12(17.1)$ & $19(14.8)$ & $23(16.2)$ & $19(22.4)$ & $27(49.1)$ & $11(55.0)$ & $<0.001$ \\
\hline Age (years) & $55.4 \pm 11.63$ & $58.0 \pm 10.47$ & $59.4 \pm 9.02$ & $60.9 \pm 8.89$ & $61.3 \pm 9.32$ & $64.0 \pm 9.72$ & $61.3 \pm 7.02$ & 0.001 \\
\hline Male & $18(54.5)$ & $42(60.0)$ & $70(60.3)$ & $93(65.5)$ & $55(64.7)$ & $33(60.0)$ & $16(80.0)$ & 0.572 \\
\hline BMI $\left(\mathrm{kg} / \mathrm{m}^{2}\right)^{\dagger}$ & $21(3.2)$ & 23.7 (3.3) & $24.2(4.2)$ & $24.8(4.4)$ & $25.8(4.2)$ & $25.9(4.1)$ & $26.3(5.8)$ & $<0.001$ \\
\hline \multicolumn{9}{|l|}{ Medication } \\
\hline$\beta$-blocker & $14(42.4)$ & $28(40.0)$ & $47(40.5)$ & $60(42.3)$ & $40(47.1)$ & $23(41.8)$ & $8(40.0)$ & 0.981 \\
\hline ACEI/ARB & $4(12.1)$ & $20(28.6)$ & $33(28.4)$ & $40(28.2)$ & $29(34.1)$ & $19(34.5)$ & $9(45.0)$ & 0.182 \\
\hline \multicolumn{9}{|l|}{ History } \\
\hline Hypertension & $8(24.2)$ & $28(40.0)$ & $48(41.4)$ & 67 (47.2) & $42(49.4)$ & $33(60.0)$ & $11(55.0)$ & 0.033 \\
\hline $\mathrm{DM}$ & $0(0.0)$ & $3(4.3)$ & $9(7.8)$ & $14(9.9)$ & $8(9.4)$ & $4(7.3)$ & $5(25.0)$ & 0.07 \\
\hline CVD & $2(6.1)$ & $7(10.0)$ & $22(19.0)$ & $29(20.4)$ & $16(18.8)$ & $19(34.5)$ & $3(15.0)$ & 0.012 \\
\hline Type of AF & & & & & & & & 0.747 \\
\hline Paroxysmal & 31 (93.9) & $64(91.4)$ & $104(89.7)$ & $128(90.1)$ & $67(78.8)$ & $38(69.1)$ & $13(65)$ & \\
\hline Persistent & $2(6.1)$ & $6(8.6)$ & $12(10.3)$ & $14(9.9)$ & $18(21.2)$ & $17(30.9)$ & $7(35)$ & \\
\hline \multicolumn{9}{|l|}{ Echocardiography } \\
\hline $\mathrm{LAD}(\mathrm{cm})$ & $2.9 \pm 0.14$ & $3.3 \pm 0.11$ & $3.6 \pm 0.12$ & $4.0 \pm 0.12$ & $4.4 \pm 0.12$ & $4.8 \pm 0.11$ & $5.3 \pm 0.13$ & $<0.001$ \\
\hline $\operatorname{LVEF}(\%)^{\dagger}$ & $68.0(4.5)$ & $67.0(6.5)$ & $66.1(6.7)$ & $64.2(8.0)$ & $62.7(8.9)$ & $63.0(8.5)$ & $63.2(11.9)$ & $<0.001$ \\
\hline LVD $(\mathrm{cm})$ & $4.7 \pm 0.37$ & $4.6 \pm 0.47$ & $4.7 \pm 0.35$ & $4.7 \pm 0.43$ & $4.8 \pm 0.45$ & $4.8 \pm 0.49$ & $4.9 \pm 0.45$ & 0.002 \\
\hline LAVe/BSA $\left(\mathrm{mL} / \mathrm{m}^{2}\right)$ & $16.6 \pm 2.9$ & $20.3 \pm 3.7$ & $25.6 \pm 5.4$ & $31.2 \pm 5.7$ & $37.8 \pm 6.2$ & $46.8 \pm 7.9$ & $54.8 \pm 9.7$ & $<0.001$ \\
\hline $\mathrm{BSA}\left(\mathrm{m}^{2}\right)$ & $1.69 \pm 0.11$ & $1.69 \pm 0.17$ & $1.79 \pm 0.18$ & $1.82 \pm 0.17$ & $1.84 \pm 0.17$ & $1.84 \pm 0.18$ & $1.89 \pm 0.13$ & 0.101 \\
\hline Anticoagulant & & & & & & & & 0.927 \\
\hline Warfarin & $23(69.7)$ & $46(65.7)$ & $76(65.5)$ & $93(65.5)$ & $56(65.9)$ & 37 (67.3) & $15(75.0)$ & \\
\hline Rivaroxaban & $9(27.3)$ & $23(32.9)$ & $35(30.2)$ & $46(32.4)$ & $24(28.2)$ & $17(30.9)$ & $5(25.0)$ & \\
\hline Dabigatran & $1(3.0)$ & $1(1.4)$ & $5(4.3)$ & $3(2.1)$ & $5(5.9)$ & $1(1.8)$ & $0(0.0)$ & \\
\hline Smoking & 8 (24.2) & $28(40.0)$ & $39(33.6)$ & $49(34.5)$ & $40(47.1)$ & $23(41.8)$ & $8(40.0)$ & 0.256 \\
\hline Alcohol consumption & $6(18.2)$ & $20(28.6)$ & $36(31.0)$ & $43(30.3)$ & 27 (31.8) & $18(32.7)$ & $7(35.0)$ & 0.824 \\
\hline Pacemaker & $1(3.0)$ & $2(2.9)$ & $3(2.6)$ & $3(2.1)$ & $5(5.9)$ & $2(3.6)$ & $1(5.0)$ & 0.718 \\
\hline
\end{tabular}

Data given as mean $\pm S D$, ${ }^{\text {median }}(\mathrm{IQR})$ or $\mathrm{n}(\%) . \mathrm{P}<0.05$ indicates statistical significance. Abbreviations as in Table 1.

\section{AF Recurrence}

The patients were divided into 7 groups on the basis of LAD in 0.4-cm increments (Table 2). With regard to the LAD subgroups (Table 2; Figure 3A), the patients with smaller and larger LAD had a higher risk of AF recurrence. The prevalence of AF recurrence reached $51.6 \%$ in patients with $\mathrm{LAD} \leq 3 \mathrm{~cm}$. The lowest proportion of $\mathrm{AF}$ recurrence was $14.8 \%$ in the patients with LAD $3.4-3.8 \mathrm{~cm}(\mathrm{P}<0.001)$. The patients with a smaller LAD were younger $(\mathrm{P}=0.002$; Table 2) and had a lower BMI $(\mathrm{P}<0.001)$. On Kaplan-Meier analysis, $\mathrm{LAD} \leq 3 \mathrm{~cm}$ or $\geq 4.6 \mathrm{~cm}$ had a significantly higher post-ablation AF recurrence rate $(\mathrm{P}<0.001$; Figure 4).

We divided the patients into 8 groups according to LAVe/BSA. Prevalence of AF recurrence reached $27.9 \%$ in patients with $\mathrm{LAVe} / \mathrm{BSA} \leq 20 \mathrm{~mL} / \mathrm{m}^{2}$. The lowest proportion of AF recurrence was $14.1 \%$ in the patients with LAVe/ BSA $25-30 \mathrm{~mL} / \mathrm{m}^{2}$. The recurrence rate in the LAVe/BSA $>50-\mathrm{mL} / \mathrm{m}^{2}$ group was $57.1 \%$ (Figure 3B). Other baseline clinical and echocardiographic pre-procedural characteristics for the subgroups are listed in Table 2.

\section{Complications}

After the first RFCA, 128 patients relapsed; 11 patients accepted a second RFCA. Seven patients had pseudoaneurysms. One patient died due to a cerebral infarction 1 month after the ablation and another patient died due to pneumonia 2 months after the ablation. Four patients had pacemakers implanted due to sinus arrest. No patients developed pericardial tamponade, pericarditis, MI, or atrial esophageal fistula after RFCA.

\section{Univariate Analysis}

The risk of AF recurrence decreased when AF was paroxysmal (HR, 0.52; 95\% CI: 0.34-0.78, $\mathrm{P}=0.002)$. LAVe/BSA (HR, 1.19; 95\% CI: 1.09-1.30, P<0.001) and LAD (HR, 1.54; 95\% CI: $1.14-2.07, \mathrm{P}=0.005$; Table 3) were also significant factors of AF recurrence after RFCA.

We defined the group with the lowest AF recurrence (LAD, $3.4-3.8 \mathrm{~cm}$ ) as the control. Compared with the control group, the unadjusted HR for AF recurrence were 3.85 (95\% CI: 2.00-7.41, P<0.001), 1.03 (95\% CI: $0.50-2.11$, $\mathrm{P}=0.945), 0.97$ (95\% CI: 0.53-1.77, $\mathrm{P}=0.907), 1.42(95 \%$ CI: $0.75-2.67, \mathrm{P}=0.285), 3.39$ (95\% CI:1.89-6.10, $\mathrm{P}<0.001)$, and $4.37(95 \% \mathrm{CI}: 2.08-9.19, \mathrm{P}<0.001)$ for $\mathrm{LAD} \leq 3 \mathrm{~cm}$, $3.0<\mathrm{LAD} \leq 3.4 \mathrm{~cm}, 3.4<\mathrm{LAD} \leq 3.8 \mathrm{~cm}, 3.8<\mathrm{LAD} \leq 4.2 \mathrm{~cm}$, $4.2<\mathrm{LAD} \leq 4.6 \mathrm{~cm}, 4.6<\mathrm{LAD} \leq 5.0 \mathrm{~cm}$, and $\mathrm{LAD}>5.0 \mathrm{~cm}$, respectively (Table 4 ).

\section{Multivariate Analysis}

On multivariate analysis, AF type (HR, 0.64; 95\% CI: $0.42-$ $0.99, \mathrm{P}=0.042)$, LAD (HR, 1.52; 95\% CI: 1.12-2.05, $\mathrm{P}=0.007)$ and LAVe/BSA (HR, 1.19; 95\% CI: 1.09-1.30, P<0.001) were independent predictors of AF recurrence (Table 4).

Compared with the control group (LAD, 3.4-3.8 cm), 


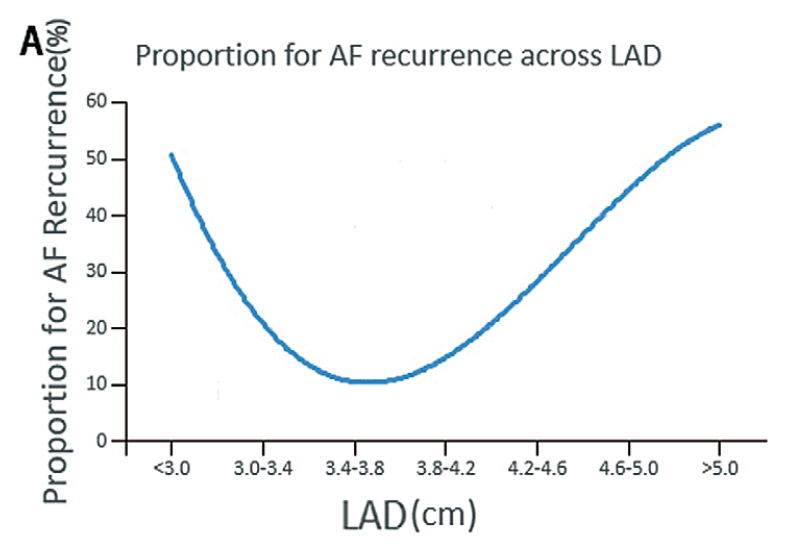

C

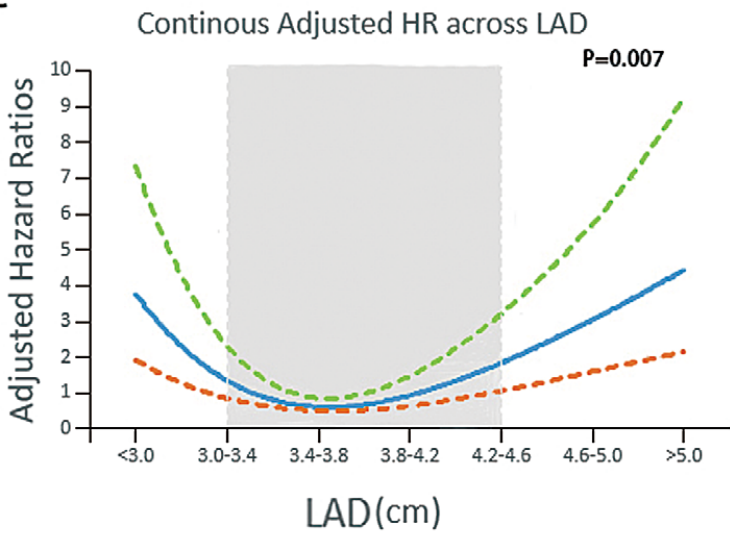

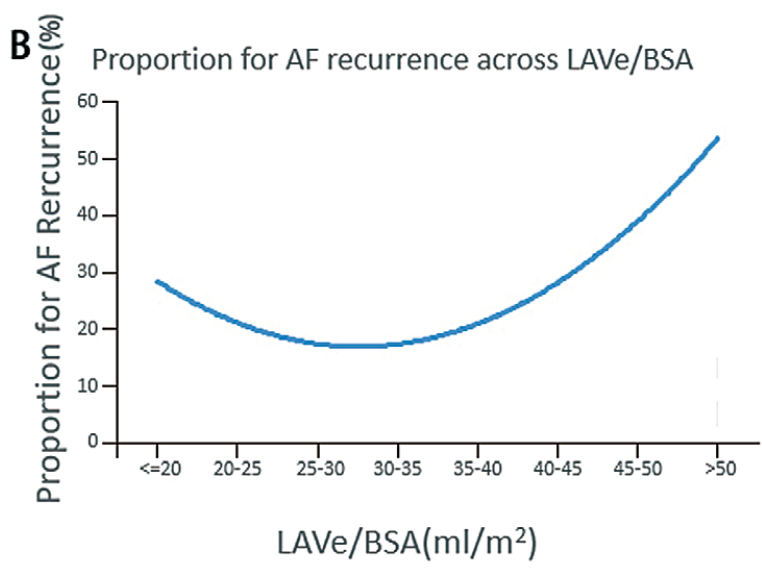

D

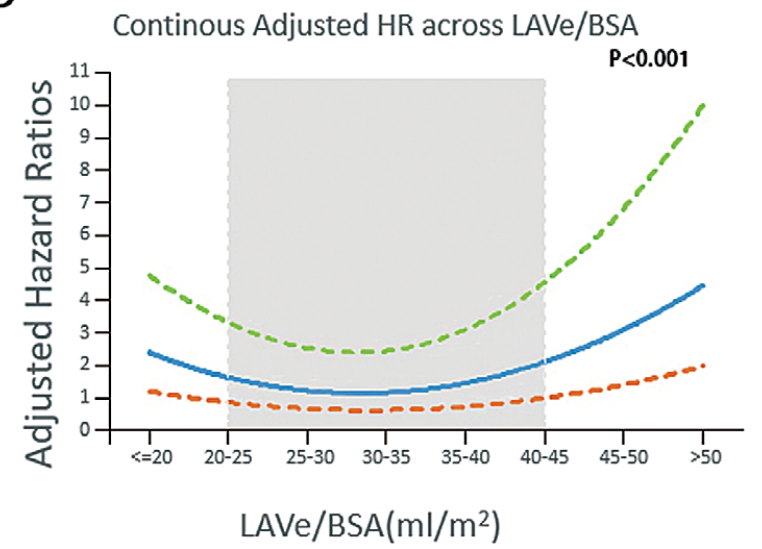

Figure 3. (A,B) Proportion of patients with atrial fibrillation (AF) recurrence after radiofrequency catheter ablation (RFCA) vs. (A) left atrium anteroposterior dimension (LAD; $P<0.001$, chi-squared test) and (B) left atrium volume (LAV) calculated using the ellipsoid model/body surface area (LAVe/BSA). (C,D) Adjusted hazard ratios (HR) for AF recurrence with 95\% Cl vs. LAD (P=0.007) and LAVe/BSA $(P<0.001)$. HR was adjusted for age, gender, diabetes mellitus, hypertension, cardiovascular disease, and type of atrial fibrillation.

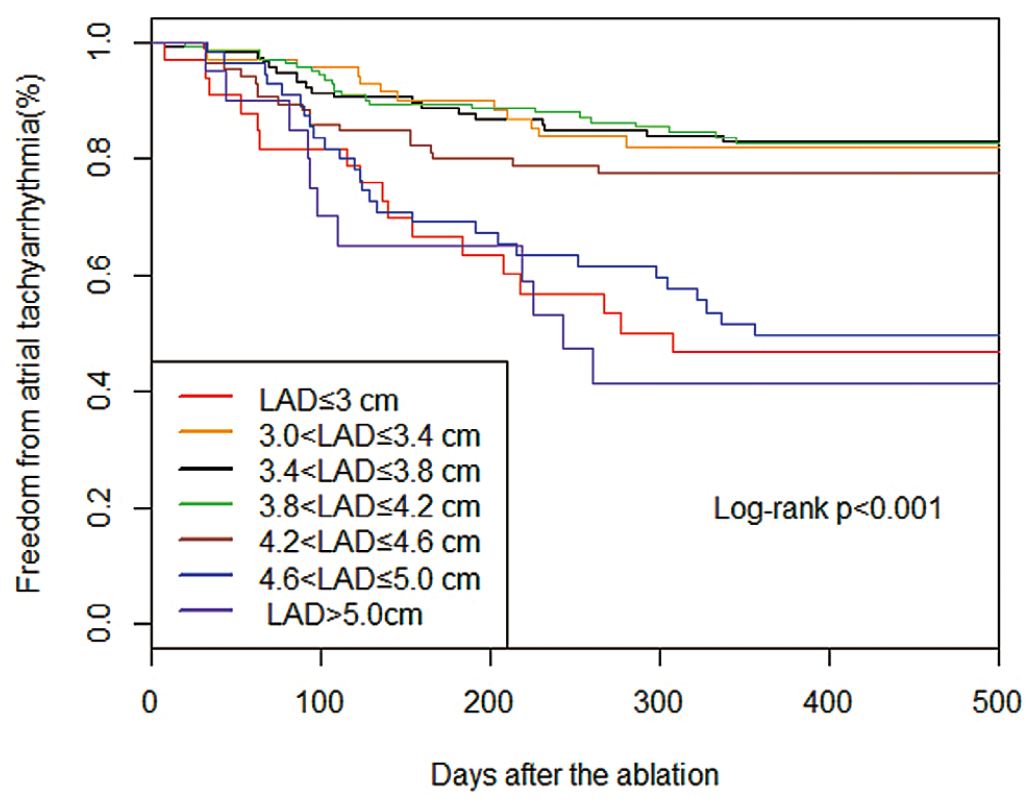

Figure 4. Kaplan-Meier cumulative eventfree survival curves after radiofrequency catheter ablation for atrial fibrillation according to left atrium anteroposterior diameter. 
the adjusted HR were 3.88 (95\% CI: 2.02-7.46, $\mathrm{P}<0.001)$, 1.03 (95\% CI: 0.50-2.12, P=0.939), 0.96 (95\% CI: 0.52-1.77, $\mathrm{P}=0.901), 1.36(95 \%$ CI: 0.72-2.57, $\mathrm{P}=0.347), 3.04(95 \%$ CI: 1.67-5.53, $\mathrm{P}<0.001)$, and 4.07 (95\% CI: 1.93-8.60, $\mathrm{P}<0.001$ ) for each group, respectively (Table 4; Figure 3C). LAD $3.0-4.6 \mathrm{~cm}$ was associated with the lowest AF recurrence. When $\mathrm{LAD}$ was $<3.0 \mathrm{~cm}$ or $>4.6 \mathrm{~cm}$, the prevalence of AF recurrence increased progressively, and followed a U-shaped curve (Figure 3A).

LAVe/BSA analysis also indicated a similar U-shaped trend. We set $\mathrm{LAVe} / \mathrm{BSA}=20-25 \mathrm{~mL} / \mathrm{m}^{2}$ as the reference. The recurrence HR were 2.31 (95\% CI: $1.08-4.94, \mathrm{P}=0.031)$, 1.68 (95\% CI: 0.79-3.56, P=0.176), 1.59 (95\% CI: 0.75-3.40, $\mathrm{P}=0.230), 1.95$ (95\% CI: $0.90-4.19, \mathrm{P}=0.089), 1.58$ (95\% CI: 0.63-3.96, $\mathrm{P}=0.328), 2.51$ (95\% CI: 1.04-6.08, $\mathrm{P}=0.041)$, and $4.95(95 \% \mathrm{CI}: 2.34-10.45, \mathrm{P}<0.001)$ for $\mathrm{LAVe} /$ $\mathrm{BSA} \leq 20 \mathrm{~mL} / \mathrm{m}^{2}, \quad 20<\mathrm{LAVe} / \mathrm{BSA} \leq 25 \mathrm{~mL} / \mathrm{m}^{2}, \quad 25<\mathrm{LAVe} /$ BSA $\leq 30 \mathrm{~mL} / \mathrm{m}^{2}, 30<\mathrm{LAVe} / \mathrm{BSA} \leq 35 \mathrm{~mL} / \mathrm{m}^{2}, 35<\mathrm{LAVe} /$ $\mathrm{BSA} \leq 40 \mathrm{~mL} / \mathrm{m}^{2}, \quad 40<\mathrm{LAVe} / \mathrm{BSA} \leq 45 \mathrm{~mL} / \mathrm{m}^{2}, 45<\mathrm{LAVe} /$ $\mathrm{BSA} \leq 50 \mathrm{~mL} / \mathrm{m}^{2}, 50 \mathrm{~mL} / \mathrm{m}^{2}<\mathrm{LAVe} / \mathrm{BSA}$, respectively, compared with $25-30 \mathrm{~mL} / \mathrm{m}^{2}$ (Table 4; Figure 3D).

\section{Discussion}

In the present study, we found that AF type (HR, 0.64; 95\% CI: $0.42-0.99, \mathrm{P}=0.042)$, LAD (HR, 1.52; 95\% CI: $1.12-2.05, \mathrm{P}=0.007)$ and LAVe/BSA (HR, 1.19; 95\% CI: $1.09-1.30, \mathrm{P}<0.001)$ were significantly associated with the prognosis of RFCA. More importantly, LA size in both the relatively larger and smaller ranges is associated with a high risk of recurrence. LAD in the $3.0-4.6-\mathrm{cm}$ and LAVe/ BSA in the $20-45-\mathrm{mL} / \mathrm{m}^{2}$ range were associated with the lowest risk for AF recurrence after RFCA. We observed a similar U-shaped curve for AF recurrence as the LA size increased, suggesting that in clinical practice, patients with an LA size that is smaller than normal may also have a

\begin{tabular}{|lcc|}
\hline \multicolumn{3}{|c|}{$\begin{array}{l}\text { Table 3. Univariate Pre-Procedure Indicators of AF } \\
\text { Recurrence }\end{array}$} \\
Age & HR (95\% Cl) & P-value \\
Male gender & $0.99(0.97-1.01)$ & 0.208 \\
CVD & $1.22(0.85-1.76)$ & 0.283 \\
Hypertension & $1.08(0.69-1.68)$ & 0.743 \\
DM & $1.30(0.91-1.85)$ & 0.145 \\
Paroxysmal AF & $1.08(0.57-2.07)$ & 0.806 \\
LAD (cm) & $0.52(0.34-0.78)$ & 0.002 \\
LVEF (\%) & $1.54(1.14-2.07)$ & 0.005 \\
LVD (cm) & $1.01(0.99-1.04)$ & 0.357 \\
LAVe/BSA (mL) & $0.90(0.59-1.36)$ & 0.616 \\
BSA & $1.19(1.09-1.30)$ & $<0.001$ \\
Pacemaker implant & $1.42(0.52-3.92)$ & 0.496 \\
BMI (kg/m $\left.{ }^{2}\right)$ & $0.64(0.28-1.45)$ & 0.281 \\
$\beta$-blocker & $1.02(0.96-1.07)$ & 0.573 \\
ACEI/ARB & $0.86(0.60-1.21)$ & 0.378 \\
Anticoagulant & $1.19(0.81-1.76)$ & 0.382 \\
Smoking & $1.15(0.66-2.01)$ & 0.615 \\
Alcohol consumption & $0.91(0.64-1.30)$ & 0.621 \\
\hline P 0.05 indicates & $0.93(0.64-1.35)$ & 0.716 \\
\hline
\end{tabular}

$\mathrm{P}<0.05$ indicates statistical significance. Abbreviations as in Table 1.

higher risk of AF recurrence.

RCFA is a more effective treatment than anti-arrhythmic drugs, ${ }^{8}$ but the therapeutic effect varies due to individual differences between patients. The success rate of RFCA is up to $80 \%$ in patients with paroxysmal AF, and as low as $75 \%$ in patients with persistent AF. 9 To improve the success rate of RFCA, a number of studies have been conducted to identify the predictors of recurrent AF, with remarkable

\begin{tabular}{|c|c|c|c|c|}
\hline & $\begin{array}{l}\text { Unadjusted HR } \\
\quad(95 \% \mathrm{Cl})\end{array}$ & P-value & $\begin{array}{l}\text { Adjusted HR }{ }^{\dagger} \\
(95 \% \mathrm{Cl})\end{array}$ & P-value \\
\hline LAD (cm) & $1.54(1.14-2.07)$ & 0.005 & $1.52(1.12-2.05)$ & 0.007 \\
\hline$L A D \leq 3.0$ & $3.85(2.00-7.41)$ & $<0.001$ & $3.88(2.02-7.46)$ & $<0.001$ \\
\hline $3.0<\mathrm{LAD} \leq 3.4$ & $1.03(0.50-2.11)$ & 0.945 & $1.03(0.50-2.12)$ & 0.939 \\
\hline $3.4<L A D \leq 3.8$ & Ref. & - & Ref. & - \\
\hline $3.8<\mathrm{LAD} \leq 4.2$ & $0.97(0.53-1.77)$ & 0.907 & $0.96(0.52-1.77)$ & 0.901 \\
\hline $4.2<\mathrm{LAD} \leq 4.6$ & $1.42(0.75-2.67)$ & 0.285 & $1.36(0.72-2.57)$ & 0.347 \\
\hline $4.6<\mathrm{LAD} \leq 5.0$ & $3.39(1.89-6.10)$ & $<0.001$ & $3.04(1.67-5.53)$ & $<0.001$ \\
\hline $5.0<\mathrm{LAD}$ & $4.37(2.08-9.19)$ & $<0.001$ & $4.07(1.93-8.60)$ & $<0.001$ \\
\hline Paroxysmal AF & $0.52(0.34-0.78)$ & 0.002 & $0.64(0.42-0.99)$ & 0.042 \\
\hline LAVe/BSA $\left(\mathrm{mL} / \mathrm{m}^{2}\right)$ & $1.19(1.09-1.30)$ & $<0.001$ & $1.19(1.09-1.30)$ & $<0.001$ \\
\hline $\mathrm{LAVe} / \mathrm{BSA} \leq 20$ & $2.21(1.03-4.71)$ & 0.041 & $2.31(1.08-4.94)$ & 0.031 \\
\hline $20<\mathrm{LAVe} / \mathrm{BSA} \leq 25$ & $1.67(0.79-3.53)$ & 0.182 & $1.68(0.79-3.56)$ & 0.176 \\
\hline $25<\mathrm{LAVe} / \mathrm{BSA} \leq 30$ & Ref. & - & Ref. & - \\
\hline $30<\mathrm{LAVe} / \mathrm{BSA} \leq 35$ & $1.64(0.77-3.49)$ & 0.204 & $1.59(0.75-3.40)$ & 0.23 \\
\hline $35<\mathrm{LAVe} / \mathrm{BSA} \leq 40$ & $1.94(0.90-4.18)$ & 0.091 & $1.95(0.90-4.19)$ & 0.089 \\
\hline $40<\mathrm{LAVe} / \mathrm{BSA} \leq 45$ & $1.80(0.72-4.47)$ & 0.206 & $1.58(0.63-3.96)$ & 0.328 \\
\hline $45<\mathrm{LAVe} / \mathrm{BSA} \leq 50$ & $2.70(1.12-6.51)$ & 0.027 & $2.51(1.04-6.08)$ & 0.041 \\
\hline $50<\mathrm{LAVe} / \mathrm{BSA}$ & $5.68(2.72-11.88)$ & $<0.001$ & $4.95(2.34-10.45)$ & $<0.001$ \\
\hline Paroxysmal AF & $0.52(0.34-0.78)$ & 0.002 & $0.56(0.36-0.89)$ & 0.013 \\
\hline
\end{tabular}

$\mathrm{P}<0.05$ indicates statistical significance. ${ }^{\dagger}$ Adjusted for age, gender, DM, hypertension, CVD, and type of AF. Abbreviations as in Table 1. 
results. ${ }^{10,11}$ The underlying mechanism of AF recurrence is still under debate. ${ }^{12}$ We showed that AF type (HR, 0.64; 95\% CI: 0.42-0.99, P=0.042) and LAD (HR, 1.52; 95\% CI: $1.12-2.05, \mathrm{P}=0.007)$ are associated with AF recurrence; another study has explored the possible mechanism. ${ }^{10}$ Atrial structure remodeling in patients with recurrent AF after RFCA has been reported in many studies. ${ }^{13,14}$ LA enlargement, which is associated with atrial remodeling, has been shown to be an independent risk factor for AF recurrence after catheter ablation. ${ }^{15}$ LAD and LA volume are commonly used to evaluate the degree of LA enlargement. The larger the LAD, the higher the rate of AF recurrence. Berruezo et al found that LAD is an independent predictor of AF recurrence, and there is a linear relationship between the increase in LAD and the risk of AF recurrence. ${ }^{6}$ Shin et al reported that LAV $34 \mathrm{~mL} / \mathrm{m}^{2}$ has a sensitivity of $70 \%$ and a specificity of $91 \%$ for predicting recurrent AF after RFCA. ${ }^{16}$ The relationship between LA enlargement and recurrent AF has been confirmed by many other studies. ${ }^{17,18}$

As noted, a considerable number of studies have shown that LAD enlargement increases the risk of AF recurrence, but no studies have shown that patients with a smaller LAD size have a higher risk of recurrent AF. We evaluated LA size using LAD and LAVe/BSA. We found that patients with smaller LA size (LAD $\leq 3.0 \mathrm{~cm}$ or $\mathrm{LAVe} / \mathrm{BSA} \leq 20 \mathrm{~mL} / \mathrm{m}^{2}$ ) had a significantly higher risk of AF recurrence compared with the control group (LAD, 3.4-3.8 cm; LAVe/BSA, $25-30 \mathrm{~mL} / \mathrm{m}^{2}$ ). In the current study, the patients in the LAD $\leq 3.0-\mathrm{cm}$ group most often had paroxysmal AF $(93.9 \%)$, and were younger than the other patients. A previous study demonstrated that $1.13 \%$ of younger people $(<50$ years of age) have AF in China. ${ }^{19}$ In the present study, most of the patients had a small LAD and were at higher risk for AF recurrence. ${ }^{19}$ Electrical reconnection of one or more PV may also play an important role in the late recurrence of AF. ${ }^{20}$ Patients with AF recurrences almost always have reconduction, and $\mathrm{PV}$ isolation may be more difficult to complete in patients with a smaller LA size. The mechanism underlying AF recurrence in these patients may be complex and warrants further research.

Race is emerging as an independent risk factor for AF. $\mathrm{AF}$ is more common in patients of European descent compared with other racial ethnic groups (i.e., African American, Chinese, and Japanese). ${ }^{21}$ This suggests that the characteristics of Chinese AF patients may be different from US and European patients. Phosphodiesterase type 4 (PDE4) is expressed in the human atrium and limits susceptibility to arrhythmia. ${ }^{22}$ The activity of PDE4 is decreased in human atrial myocytes with persistent AF.23 Phosphodiesterase inhibitors (PDEI) have been suggested as the optimal treatment for Asian people with heart failure, but PDEI are contraindicated in Western patients. ${ }^{24}$ The differing efficacy of PDEI in Asian and Western patients has led some researchers to believe that PDE activity may vary with race and may lead to the heterogeneity in the outcomes of AF recurrence. ${ }^{23}$ Genetic variants may be another factor in the differing prognosis after RFCA. ${ }^{25}$ Because the characteristics of Chinese AF patients are different from those in the USA and Europe, the present results may not be generalizable. There are no articles in Europe or the USA, however, on the relationship between smaller LA and AF recurrence. Therefore, more studies are needed to further elucidate the role of smaller LA size in the recurrence of AF in these patients.

\section{Study Limitations}

This study had several limitations. First, the patients who had persistent AF were few in number. Second, as noted here, the characteristics of Chinese AF patients differ to those in patients from the USA and Europe, and the results may not be generalizable. Thus, the true relationship between LA size and AF recurrence warrants further research. Third, in the present study routine follow-up was performed with 48-h Holter monitors, which are not as accurate as 1-2week monitors or injectable loop recorders, and may lead to ascertainment bias.

\section{Conclusions}

Patients with LAD in the range $3.0-4.6 \mathrm{~cm}$ and LAVe/BSA $20-45 \mathrm{~mL} / \mathrm{m}^{2}$ were at the lowest risk for AF recurrence 1 year after RFCA. Patients with a smaller or larger LA size were at higher risk for AF recurrence. The relationship between LA size and AF recurrence 1 year after RFCA followed a U-shaped curve. This indicates that patients with a small LAD and also LAVe/BSA have a high incidence of AF recurrence, and need to be managed accordingly.

\section{Acknowledgments}

This study was supported by the National Science Foundation for Young Scholars of China (grant nos. 30900612 and 81800231), the National Science Foundation of China (grant no. 81873484), the Clinical Research Fund of Zhejiang Provincial Medical Association (2015ZYC-A16 and 2018ZYC-A11), the Nature Science Foundation of Zhejiang Province (grant no. LZ16H020001), the Medical Science Research Foundation of Zhejiang Province (grant no. 2018ZD017), the Zhejiang Provincial Natural Science Foundation (grant no. LY15H020002 and Y17H020020), and the Department of Science and Technology, Zhejiang Province (grant no. LGF19H020011), People's Republic of China.

\section{Disclosures}

The authors declare no conflicts of interest.

\section{References}

1. Rahman F, Kwan GF, Benjamin EJ. Global epidemiology of atrial fibrillation. Nat Rev Cardiol 2014; 11: 639-654.

2. $\mathrm{Hu} \mathrm{D}$, Sun Y. Epidemiology, risk factors for stroke, and management of atrial fibrillation in China. $J$ Am Coll Cardiol 2008; 52: $865-868$.

3. January CT, Wann LS, Alpert JS, Calkins H, Cigarroa JE, Cleveland JC Jr, et al. 2014 AHA/ACC/HRS guideline for the management of patients with atrial fibrillation: A report of the American College of Cardiology/American Heart Association Task Force on Practice Guidelines and the Heart Rhythm Society. J Am Coll Cardiol 2014; 64: 2305-2307.

4. Weerasooriya R, Khairy P, Litalien J, Macle L, Hocini M, Sacher F, et al. Catheter ablation for atrial fibrillation: Are results maintained at 5 years of follow-up? J Am Coll Cardiol 2011; 57: 160-166.

5. Zhuang J, Wang Y, Tang K, Li X, Peng W, Liang C, et al. Association between left atrial size and atrial fibrillation recurrence after single circumferential pulmonary vein isolation: A systematic review and meta-analysis of observational studies. Europace 2012; 14: 638-645.

6. Berruezo A, Tamborero D, Mont L, Benito B, Tolosana JM, Sitges M, et al. Pre-procedural predictors of atrial fibrillation recurrence after circumferential pulmonary vein ablation. Eur Heart J 2007; 28: 836-841.

7. Lang RM, Badano LP, Mor-Avi V, Afilalo J, Armstrong A, Ernande L, et al. Recommendations for cardiac chamber quantification by echocardiography in adults: An update from the American Society of Echocardiography and the European Association of Cardiovascular Imaging. Eur Heart $J$ Cardiovasc Imaging 2015; 16: 233-270.

8. Wazni OM, Marrouche NF, Martin DO, Verma A, Bhargava M, Saliba W, et al. Radiofrequency ablation vs antiarrhythmic drugs 
as first-line treatment of symptomatic atrial fibrillation: A randomized trial. JAMA 2005; 293: 2634-2640.

9. Cappato R, Calkins H, Chen SA, Davies W, Iesaka Y, Kalman $\mathrm{J}$, et al. Updated worldwide survey on the methods, efficacy, and safety of catheter ablation for human atrial fibrillation. Circ Arrhythm Electrophysiol 2010; 3: 32-38.

10. Vasamreddy CR, Lickfett L, Jayam VK, Nasir K, Bradley DJ, Eldadah Z, et al. Predictors of recurrence following catheter ablation of atrial fibrillation using an irrigated-tip ablation catheter. J Cardiovasc Electrophysiol 2004; 15: 692-697.

11. Sultan A, Lüker J, Andresen D, Kuck KH, Hoffmann E, Brachmann J, et al. Predictors of atrial fibrillation recurrence after catheter ablation: Data from the German Ablation Registry. Sci Rep 2017; 7: 16678.

12. Balk EM, Garlitski AC, Alsheikh-Ali AA, Terasawa T, Chung M, Ip S. Predictors of atrial fibrillation recurrence after radiofrequency catheter ablation: A systematic review. J Cardiovasc Electrophysiol 2010; 21: $1208-1216$.

13. Sung SH, Chang SL, Hsu TL, Yu WC, Tai CT, Lin YJ, et al. Do the left atrial substrate properties correlate with the left atrial mechanical function? A novel insight from the electromechanical study in patients with atrial fibrillation. J Cardiovasc Electrophysiol 2008; 19: $165-171$.

14. Avitall B, Bi J, Mykytsey A, Chicos A. Atrial and ventricular fibrosis induced by atrial fibrillation: Evidence to support early rhythm control. Heart Rhythm 2008; 5: 839-845.

15. den Uijl DW, Delgado V, Bertini M, Tops LF, Trines SA, van de Veire NR, et al. Impact of left atrial fibrosis and left atrial size on the outcome of catheter ablation for atrial fibrillation. Heart 2011 97: $1847-1851$

16. Shin SH, Park MY, Oh WJ, Hong SJ, Pak HN, Song WH, et al. Left atrial volume is a predictor of atrial fibrillation recurrence after catheter ablation. J Am Soc Echocardiogr 2008; 21: $697-$ 702.

17. Arya A, Hindricks G, Sommer P, Huo Y, Bollmann A, Gaspar T, et al. Long-term results and the predictors of outcome of catheter ablation of atrial fibrillation using steerable sheath catheter navigation after single procedure in 674 patients. Europace 2010; 12: $173-180$.

18. Tang RB, Yan XL, Dong JZ, Kalifa J, Long DY, Yu RH, et al. Predictors of recurrence after a repeat ablation procedure for paroxysmal atrial fibrillation: Role of left atrial enlargement. Europace 2014; 16: 1569-1574.

19. Wang X, Fu Q, Song F, Li W, Yin X, Yue W, et al. Prevalence of atrial fibrillation in different socioeconomic regions of China and its association with stroke: Results from a national stroke screening survey. Int J Cardiol 2018; 271: 92-97.

20. Kuck KH, Hoffmann BA, Ernst S, Wegscheider K, Treszl A, Metzner A, et al. Impact of complete versus incomplete circumferential lines around the pulmonary veins during catheter ablation of paroxysmal atrial fibrillation: Results from the Gap-Atrial Fibrillation-German Atrial Fibrillation Competence Network 1 Trial. Circ Arrhythm Electrophysiol 2016; 9: 003337.

21. Leef GC, Perino AC, Cluckey A, Yunus FN, Askari M, Heidenreich PA, et al. Geographic and racial representation and reported success rates of studies of catheter ablation for atrial fibrillation: Findings from the SMASH-AF meta-analysis study cohort. J Cardiovasc Electrophysiol 2018; 29: 747-755.

22. Molina CE, Leroy J, Richter W, Xie M, Scheitrum C, Lee IO, et al. Cyclic adenosine monophosphate phosphodiesterase type 4 protects against atrial arrhythmias. J Am Coll Cardiol 2012; 59: $2182-2190$.

23. Lau CP, Tse HF, Siu CW, Gbadebo D. Atrial electrical and structural remodeling: Implications for racial differences in atrial fibrillation. J Cardiovasc Electrophysiol 2012; 23 (Suppl 1): S36S40.

24. Sasayama S. Heart disease in Asia. Circulation 2008; 118: $2669-$ 2671

25. Gudbjartsson DF, Holm H, Gretarsdottir S, Thorleifsson G, Walters GB, Thorgeirsson G, et al. A sequence variant in ZFHX3 on 16q22 associates with atrial fibrillation and ischemic stroke. Nat Genet 2009; 41: 876-878. 